\title{
IMPROVEMENT ON TIMING ACCURACY OF LIDAR FOR REMOTE SENSING
}

\author{
Guoqing Zhou ${ }^{1}$, Wei Huang ${ }^{1,2}$, Xiang Zhou ${ }^{1,2, *}$, Yu Huang ${ }^{1}$, Chaoshuang He ${ }^{1}$, Xiaozhu Li ${ }^{1}$, Lieping Zhang ${ }^{1,2}$ \\ ${ }^{1}$ Guangxi Key Laboratory of Spatial Information and Geomatics, Guilin University of Technology, No. 12 Jian'gan Road, Guilin, \\ Guangxi 541004, China - gzhou@glut.edu.cn \\ 2 Department of Mechanical and Control Engineering, Guilin University of Technology, No. 12 Jian'gan Road, Guilin, Guangxi \\ 541004, China - (102016477, zqx0711)@glut.edu.cn
}

\section{Commission III, ICWG III/IVb}

KEY WORDS: Laser rangefinding, Leading-edge timing discrimination, Walk error, MATLAB, Lidar data quality

\begin{abstract}
:
The traditional timing discrimination technique for laser rangefinding in remote sensing, which is lower in measurement performance and also has a larger error, has been unable to meet the high precision measurement and high definition lidar image. To solve this problem, an improvement of timing accuracy based on the improved leading-edge timing discrimination (LED) is proposed. Firstly, the method enables the corresponding timing point of the same threshold to move forward with the multiple amplifying of the received signal. Then, timing information is sampled, and fitted the timing points through algorithms in MATLAB software. Finally, the minimum timing error is calculated by the fitting function. Thereby, the timing error of the received signal from the lidar is compressed and the lidar data quality is improved. Experiments show that timing error can be significantly reduced by the multiple amplifying of the received signal and the algorithm of fitting the parameters, and a timing accuracy of 4.63 ps is achieved.
\end{abstract}

\section{INTRODUCTION}

Pulsed lidar is currently the most popular measurement tool in remote sensing applications, especially for ranging and 3D imaging (Gao, 2014; Zhou, 2004, 2009, 2014 and 2015). The key parameters of it are the range and the accuracy. The range mainly depends on the energy of the emitted pulse, and there are many parameters that determine the measurement accuracy. For example, the Experimental environment, waveform of received signal, the accuracy of time-interval and timing discrimination. Generally, the waveform of received signal is affected by the environment, and the accuracy of the timeinterval is determined by the timing discrimination. Therefore, the timing discrimination is one of the most important steps in determining the accuracy of pulsed lidar for remote sensing.

The classical methods of timing include LED (Gottschalk, 1983; Peltola, 2000; Zhou, 2017), constant fraction timing (Zheng, 2012; Cheng, 2015) and high-pass filtering timing (Kurtti, 2009; Jiang, 2015). The LED is widely used due to the simple circuit structure, easy operation and fast signal processing, but the measurement accuracy is not high. Constant fraction timing and high-pass filtering timing with high accuracy, but circuit is complex, and the received signal is demanded. Many efforts have been made on the accuracy improvement of LED. Gupta (2005) designed a simple circuit to convert the output signal of PMT to digital pulse, which can achieve a timing error of 90 ps under in the actual environment. Lage (2010) proposed and evaluated a hybrid analog/digital scheme that achieves a timing accuracy of $1.32 \mathrm{~ns}$ at a rise time of $1.5 \mathrm{~ns}, 6 \mathrm{~ns}$ width and 800 $\mathrm{mV}$ amplitude. Kurtti (2012) measured the length of the received pulse based on multi-channel time digital conversion circuit (TDC), and used the known relationship to compensate for the walk error (Kurtti, 2009); the minimum error was $2.2 \mathrm{~ns}$.
A timing point sampling with multi-level amplification for received signal based on LED is presented in this paper. The function of the amplifying factor and the corresponding timing point that combined with fitting algorithm is obtained in MATLAB software. The maximum error limit is set according to the ranging requirement, and the minimum error range is obtained by the fitting function. Therefore, the walk error is reduced and the quality of the lidar data is improved.

\section{PRINCIPLE OF RECEIVING CHANNEL}

\subsection{General Principle}

The receiving system of pulsed lidar mainly consists of receiving optical unit, photoelectric conversion unit, preamplifier unit, timing discrimination unit, time-interval measuring unit and control unit. The receiving system is shown in Figure 1. The principle is based on the time of flight (Palojarvi, 1996), that is, the round-trip time between the start signal and the stop signal, and then sends this information to the controller which calculates the corresponding spatial distance. In the traditional LED, since the threshold is compared with the received signal only once to determine the timing point and different timing points due to a different threshold, which means different timing errors (Figure 2).

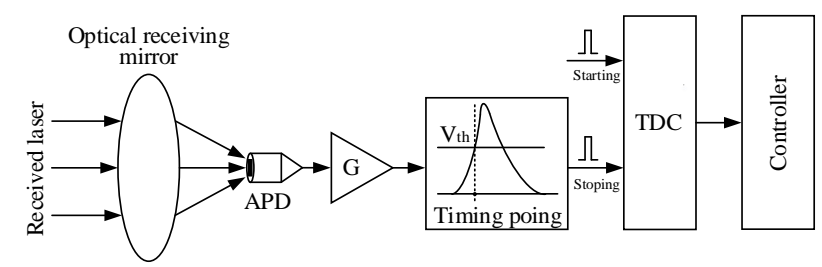

Figure 1. The framework of the receiving system

\footnotetext{
* Corresponding author: Xiang Zhou; E-mail: zqx0711@glut.edu.cn
} 


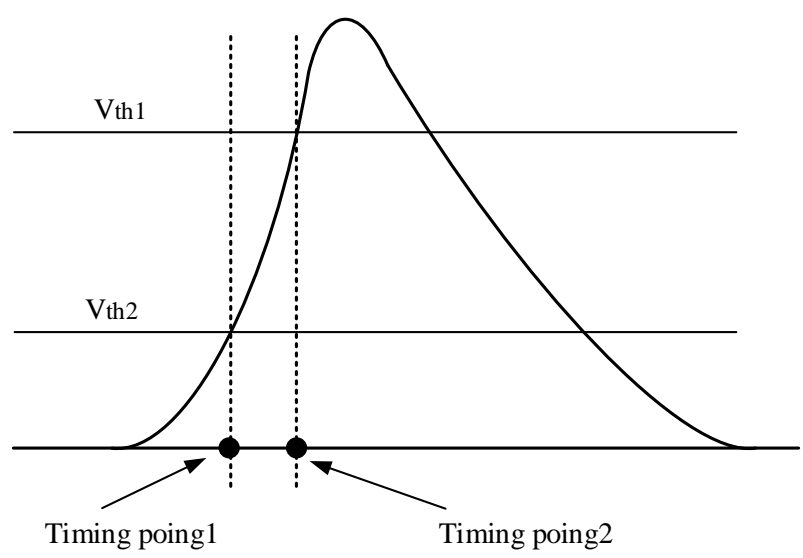

Figure 2. The principle of LED based on different threshold

\subsection{Principle Based on Multiple Amplification and Fitting}

The principle of timing based on amplification and fitting algorithm can be understood as follows. First of all, access to relevant information. The received signal is amplified in parallel, and the peak value is obtained by the A/D sampling circuit and sent to the control unit; the threshold is set to be fixed and each pulse signal for quick comparison, and obtains the corresponding timing point information. Then, the collected information is calculated. All the timing information is sent to the TDC unit and a plurality of time-intervals is generated and sent to the control unit. In the control unit, all the information of peak points and corresponding timing points are plotted in MATLAB software together, and then its functional relationship are found. Finally, predict the walk error based on the fitted curve. Through the mechanism of data post-processing, the timing point is infinitely close to the beginning of the received signal. The specific operation is shown in Figure 3. In order to achieve curve fitting, at least three timing points are needed. The peak value of the original received signal is set to be $A_{0}$, the timing point is $t_{0}$, the amplification factors are $G_{1}, G_{2}$, $G_{3}, \cdots, G_{\mathrm{n}}$, the signal peaks amplified step by step are $A_{1}, A_{2}$, $A_{3}, \cdots, A_{\mathrm{n}}$, the corresponding timing points are $t_{1}, t_{2}, t_{3}, \cdots, t_{\mathrm{n}}$. Then, the peak value of signal at different amplification factors and the corresponding timing point information are as

$$
X=\left[\begin{array}{cccccc}
A_{0} & A_{1} & A_{2} & A_{3} & \cdots & A_{n} \\
- & G_{1} & G_{2} & G_{3} & \cdots & G_{n} \\
t_{0} & t_{1} & t_{2} & t_{3} & \cdots & t_{n}
\end{array}\right]
$$

where $A_{0}=$ original peak value

$A_{1}, \cdots, A_{\mathrm{n}}=$ amplified peak values

$G_{1}, \cdots, G_{\mathrm{n}}=$ amplification factors

$t_{0}, \cdots, t_{\mathrm{n}}=$ timing point

In the above relation, the magnification factor is calculated from the second measurement. Furthermore, the calculated amplification factors are all compared with the magnitude of the first measurement.

As shown in Figure 3, the method of LED is independent of the amplitude range, and the linear amplification of the received signal can be over a large dynamic range in the receive channel $(1: 1000 \cdots 000)$. As the receiving pulse signal increased gradually, the intersection of the rising edge and the threshold continuously moves forward $\left(t_{0}>t_{1}>t_{2}>t_{3}>\ldots>t_{\mathrm{n}}\right)$. Theoretically, the rising edge tends to be vertical as it enlarges gradually, and the minimum timing point lies at the intersection of the rising edge and $\mathrm{V}_{\mathrm{th}}$. Due to the complexity of the actual circuit and noise interference, these are very hard to achieve. However, it is possible to obtain the timing point of the minimum error with the rising edge approximately vertical.

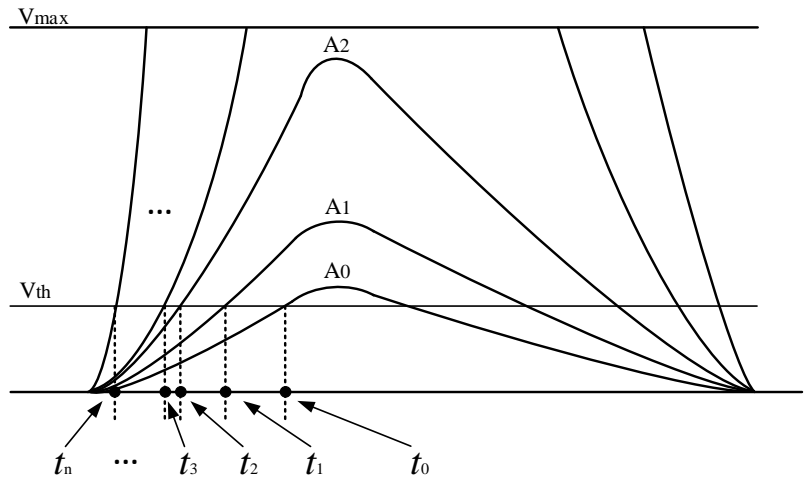

Figure 3. The principle of LED based on multiple amplification

In order to obtain the minimum timing within the allowable range of error, Based on the study of $X$, the inline relationship between walk error and amplification factor and peak value of the received signal is obtained, and the minimum walk error is deduced. So, an optimal fitting algorithm is needed. In the CFTOOL toolbox of MATLAB software (MATLAB Development Team, 2016), the main fit models are Custom Equation, Exponential, Fourier, Gaussian, Interpolant, Linear Fitting, Polynomial, Power, Rational, Smoothing Spline, Sum of Sine and Weibull. According to the analysis of the timing point and the amplification factor and peak value, it is clear that different amplification factors have different peak values, which also have different timing points. Through the analysis of data in Table 1, we can see that the relationship between the peak value and the timing point is a change in the opposite direction, that is, the larger the peak values, the timing points will gradually decrease. This function relation is a change curve with decreasing rate. Therefore, fitting models that initially meet the data processing requirements are Power and Exponential. In the model of Power, the general formula used is

$$
f=a \cdot x^{b}+c
$$

$$
\text { where } \begin{aligned}
f & =\text { dependent variable } \\
x & =\text { independent variable } \\
a & =\text { weight of the independent variable plus power } \\
b & =\text { power } \\
c & =\text { compensation constant }
\end{aligned}
$$

In formula $2, x$ corresponds to different peak values, and $f$ corresponds to different timing points. By reasonably choosing the values of $a, b$ and $c$, the timing points in Table 1 fall on the same curve completely. The final actual curve function can be considered as the relation of the different timing points corresponding to all the peaks and the amplification factors. In the model of Exponential (second order), the general formula used is

$$
f=a \cdot e^{b x}+c \cdot e^{d x}
$$

where $f=$ dependent variable

$x=$ independent variable

$a, b, c, d=$ corresponding coefficient 
In this model, the peak value is the index variable, which will make the curve model approach to all the points in Table 1, but this model cannot get the extreme value, because there is no compensation constant. That is, when the peak value is infinite to infinity, a valid timing point cannot be obtained. Although this model can perfect the fitting of the sampling points, it cannot meet the final extremum evaluation, so it is abandoned.

\section{EXPERIMENT AND ANALYSIS}

\subsection{Measurement and Results}

The experiment was completed based on pulsed lidar in laboratory. The avalanche photodiode (APD) (Albota, 2002) produced by Pacific Silicon Sensor and the $905 \mathrm{~nm}$ AD500-8TO52S2 near-red light emitter of Laser Components were selected. The avalanche current output from APD through the transimpedance into a voltage pulse signal range of $10 \mathrm{mV}$ to $1 \mathrm{~V}$ and dark current is less than $6 \mathrm{nA}$. In order to verify the proposed scheme more simply, the experiment made some simplifications. The laser emitters directly emit pulse signals of the same pulse width and different amplitudes to APD detectors. Experimental platform was built as shown in Figure 4. The transmitter signal is amplified step-by-step through a laser transmitter (12 mV to $895 \mathrm{mV}$ ) and the threshold of comparator is set to $8 \mathrm{mV}$. A series of data was measured as shown in Table 1 .

\begin{tabular}{|l|cccc|}
\hline Peak Value (mV) & 12 & 78 & 177 & 263 \\
Amplification factor & - & 6.5 & 14.75 & 21.92 \\
Timing Point (ps) & 2300.254 & 324.985 & 142.496 & 96.493 \\
\hline Peak Value (mV) & 361 & 438 & 527 & 636 \\
Amplification factor & 30.08 & 36.5 & 43.92 & 53 \\
Timing Point (ps) & 70.002 & 57.987 & 47.951 & 39.679 \\
\hline Peak Value (mV) & 744 & 819 & 895 & \\
Amplification factor & 62 & 68.25 & 74.58 & \\
Timing Point (ps) & 34.251 & 30.995 & 28.288 & \\
\hline
\end{tabular}

Table 1 . The characteristic parameters of the received signal

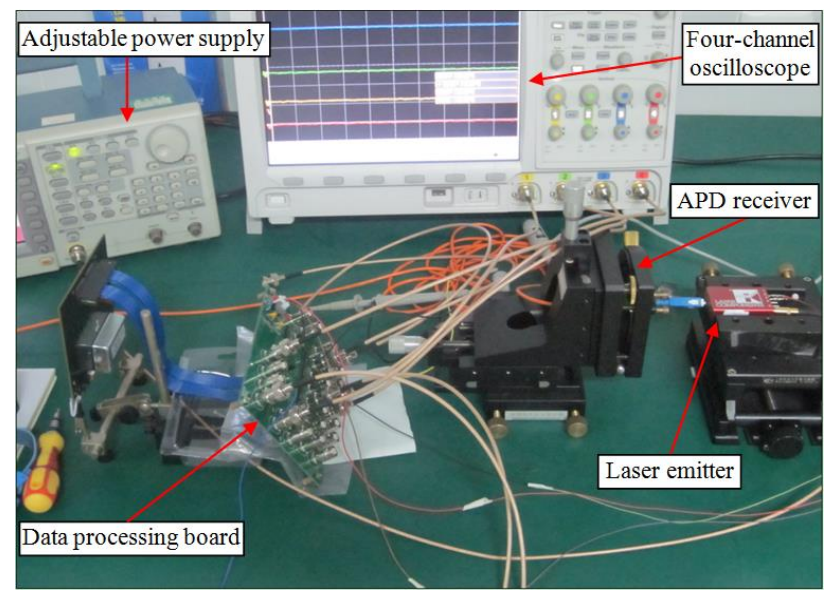

Figure 4. The measurement platform in the laboratory

\subsection{Error Evaluation}

According to formulas (1) and (2), the data of Table 1 is imported into MATLAB software, and the sampling peak point (Sp) and the sampling timing point (St) of the signal, CFTOOL toolbox in MATLAB software is used, and the general model Power 2 is selected to fit the data, and the fitting curve is obtained (Figure 5). According to the results of the fitting in Figure 5, the goodness of fit test results are as follows: the sum of squares due to error is 13.21 , the R-square is 1 and the RMSE is 1.285. That means the deviation of the fitting result can be neglected when compared with the actual situation, and can be regarded as a real relationship. According to the fitting function with $95 \%$ confidence bounds is as

$$
f=3.144 \times 10^{4} \cdot x^{-1.049}+4.63
$$

According to formula (4), the timing points of the received signals are estimated. It can be seen that with the amplification of the received signal, the walk error will gradually decrease. But this trend will not become obvious with the increase of amplification factor, and an appropriate point can be selected as the timing point to evaluate the ranging accuracy of lidar (a kind of tends to infinity, timing point is $4.63 \mathrm{ps}$ ). In order to verify the correctness of the fitting function, an accuracy evaluation is shown in Figure 6. An additional 34 sampling peak points will be compared with the calculation of formula (4). The results show that the error between the calculated result and the actual measured value does not exceed 4.63 ps according to the formula Figure 6, which fully proves the correctness and practicability of the fitting model. Additionally, the experimental accuracy of proposed method has compared with the accuracy of other methods of LED (Table 2), and the walk error decreases over $60 \%$.

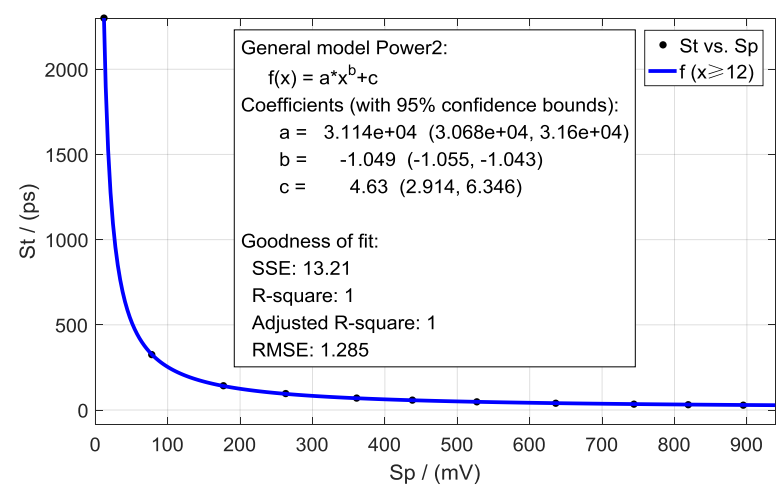

Figure 5. The relationship between different peaks and timing points with the general model Power2

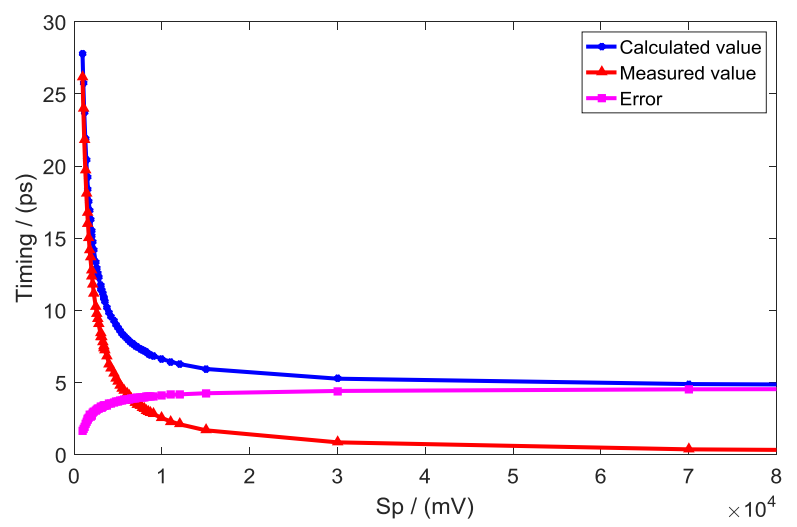

Figure 6. Accuracy evaluation of timing point for fitting function 


\begin{tabular}{|l|c|c|}
\hline Methods & $\begin{array}{c}\text { Sampling times of } \\
\text { timing points }\end{array}$ & $\begin{array}{c}\text { Minimum walk } \\
\text { error }(\mathrm{ps})\end{array}$ \\
\hline Gupta (2005) & 1 & 1320 \\
Lage (2010) & 1 & 90 \\
Kurtti (2012) & 4 & 2200 \\
Our method & 11 & 4.63 \\
\hline
\end{tabular}

Table 2. The comparison of experimental accuracy for other timing discrimination with LED

\section{CONCLUSION}

In this paper, a LED based on multiple amplifying of received signal and the fitting algorithm of timing information in pulsed lidar is proposed. Fitting algorithm is performed with the parameters of peak value, amplification factor and timing point. A relationship based on these factors are found, and verified to be correct and practical by comparison with actual measurements. The minimum timing walk error is obtained based on the model of fitting function. Compared with other LED, the proposed method has a higher accuracy. The minimum walk error is less than 5 ps and the walk error decreases over $60 \%$. Furthermore, this work can provide a useful reference for the timing discrimination of pulsed lidar in remote sensing.

\section{ACKNOWLEDGEMENTS}

This paper is financially supported by the National Key Research and Development Program of China under Grant numbers 2016YFB0502500, the National Natural Science of China under Grant numbers 41431179, the State Oceanic Administration under Grant numbers 2014\#58, GuangXi Natural Science Foundation under Grant numbers 2015GXNSFDA139032, Guangxi Science \& Technology Development Program under the Contract numbers GuiKeHe 14123001-4, GuangXi Key Laboratory of Spatial Information and Geomatics Program under Grant numbers 151400701, 151400712 and 16380251, Guangxi Young and Middle-aged Teachers Basic Ability Improvement Project under Grant numbers 2018KY0238, Guangxi doctoral student innovation under Grant numbers YCBZ2018054.

\section{REFERENCES}

Albota, M.A., Heinrichs, R.M., Kocher, D.G., Fouche, D.G., Player, B.E., O’Brien, M.E., Aull, B.F., Zayhowski, J.J., Mooney, J., Willard, B.C. and Carlson, R.R., 2002. Threedimensional imaging laser radar with a photon-counting avalanche photodiode array and microchip laser. Applied optics. 41(36), 7671.

Cheng, Z., Yang, K., Han, J., Zhou, Y.,Sun, L., Li, W. and Xia, M., 2015. Improved time-of-flight range acquisition technique in underwater lidar experiments. Appl. Optics. 54, pp. 57155725 .

Gao, C.X. and Niu, L.Q., 2014. The pulsed all fiber laser application in the high-resolution 3D imaging LIDAR system. Laser Sources \& Applications II. 9135(3), 91351M.

Gottschalk, B., 1983. Timing discriminator using leading-edge extrapolation. Nuclear Instruments \& Methods in Physics Research. 190(1), pp. 67-70.
Gupta, S.K., Hayashi, Y., Jain, A., Karthikeyan, S., kawakami, S., Ravindran, K.C. and Tonwar, S.C., 2005. A highperformance, low-cost, leading edge discriminator. Pramana. 65(2), pp. 273-283.

Jiang, Y., Liu, R. and Zhu, J., 2015. Integrated multi-channel receiver for a pulsed time-of-flight laser radar. In Proceedings of Photoelectronic Technology Committee Conferences. International Society for Optics and Photonics. 95220C.

Kurtti, S. and Kostamovaara, J., 2009. Laser Radar Receiver Channel With Timing Detector Based on Front End Unipolarto-Bipolar Pulse Shaping. IEEE Journal of Solid-State Circuits. 44(3), pp. 835-847.

Kurtti, S. and Kostamovaara, J., 2012. An integrated receiver channel for a laser scanner. Instrumentation and Measurement Technology Conference, IEEE. 8443, pp. 1358-1361.

Lage, E., Tapias, G., Villena, J., Desco, M. and Vaquero, J.J., 2010. Data acquisition electronics for gamma ray emission tomography using width-modulated leading-edge discriminators. Physics in Medicine \& Biology. 55(15), pp. 4291-4308.

MATLAB Development Team, 2016. Matrix Laboratory (MATLAB) Software, Version 9.0. Business Mathematics Software https://cn.mathworks.com (10 December 2017)

Peltola, T., Ruotsalainen, T., Palojarvi, P. and Kostamovaara, J, 2000. A receiver channel with a leading edge timing discriminator for a pulsed time-of-flight laser radar. Solid-State Circuits Conference, 2000. Esscirc '00. Proceedings of the, European. IEEE. pp. 427-430.

Palojarvi, P., Maatta, K. and Kostamovaara, J., 1996. Integrated time-of-flight laser radar. IEEE Transactions on Instrumentation \& Measurement. 46(4), pp. 996-999.

Zheng, R. and Guanhao, W.U., 2012. Constant fraction discriminator in pulsed time-of-flight laser rangefinding. Frontiers of Optoelectronics. 5(2), pp. 182-186.

Zhou, G., Song, C. and Schickler, W., 2004. Urban 3D GIS from LIDAR and aerial image data. Computers and Geosciences, 30(4), pp. 345-353.

Zhou, G. and Xie, M., 2009. GIS-based Three-dimensional Morphologic Analysis of Assateague Island National Seashore from LIDAR Series Datasets. Journal of Coastal Research, 25(2), pp. 435-447.

Zhou, G., and Zhou. X., 2014. Seamless Fusion of LiDAR and Aerial Imagery for Building Extraction. IEEE Transactions on Geoscience and Remote Sensing, 52(11), pp. 7393-7407.

Zhou, G., Zhou, X., Yang, J., Tao, Y., Nong, X. and Baysal, O., 2015. Flash Lidar Sensor using Fiber Coupled APDs. IEEE Sensor Journal, 15(9), pp. 4758-4768.

Zhou, G., Huang, W., Zhou, X., Zhang, L., Chen, P., Huang, J. and Zhang, R., 2017. A new approach to minimize walk error in pulsed laser rangefinding. IGARSS 2017 - 2017 IEEE International Geoscience and Remote Sensing Symposium. IEEE, pp. 1708-1711. 